\title{
Edge surface supercurrent and transport anomalies in Hall effect measurements in $\mathrm{Nb}$
}

\section{Dan Zhou}

Soochow University

Hansong Zeng

Institute for Advanced Study

Rujun Tang

Soochow University

Zhi Hong Hang

Soochow University

Zhiwei Hu

Institute of Physics

Zixi Pei

Institute of Physics

Xinsheng Ling ( $\nabla$ xinsheng_ling@brown.edu )

Brown University

\section{Article}

Keywords: superconductivity, Hall effect, edge surface supercurrent

Posted Date: March 29th, 2021

DOI: https://doi.org/10.21203/rs.3.rs-373542/v1

License: (c) (i) This work is licensed under a Creative Commons Attribution 4.0 International License.

Read Full License 


\title{
Edge surface supercurrent and transport anomalies in Hall effect measurements in $\mathrm{Nb}$
}

\author{
Dan Zhou ${ }^{1}$, Hansong Zeng ${ }^{1}$, Rujun Tang ${ }^{1, *}$, Zhi H. Hang ${ }^{1}$, Zhiwei Hu ${ }^{2}$, Zixi Pei ${ }^{2}$, and \\ Xinsheng Sean Ling ${ }^{3, *}$
}

${ }^{1}$ Institute for Advanced Study, School of Physical Science and Technology, Soochow University, Suzhou, 215006, People's Republic of China

${ }^{2}$ Institute of Physics, Chinese Academy of Science, Beijing, 100190, P.R. China

${ }^{3}$ Department of Physics, Brown University, Providence, Rhode Island 02912, USA

*corresponding to: xinsheng_ling@brown.edu; tangrj@suda.edu.cn

\begin{abstract}
We report an experimental study of the edge surface superconductivity and the Hall effect in a $\mathrm{Nb}$ thin film Hall device. We show that the previously reported anomalous sign reversal of the Hall resistance across the superconducting-normal transition is due to the edge supercurrent. Large anomalous oscillations between the transverse voltage probes are observed in the field range between $H_{c 2}$ and $H_{c 3}$, in both the anti-symmetric (Hall) and symmetric (resistive) channels. These oscillations are extremely sensitive to a small tilting of the edge surface relative to the external magnetic field. We suggest that these oscillations may arise from the Aharonov-Bohm interference of the edge supercurrents.
\end{abstract}

\section{Introduction}

Saint-James and de Gennes (SJdG) showed ${ }^{1}$ that, within the Ginzburg-Landau theory of superconductivity ${ }^{2}$, there exists the possibility of a novel phenomenon of surface superconductivity, that with decreasing magnetic field, at a constant temperature below the superconducting transition temperature $T_{c}$, the superconducting order parameter $\psi$ becomes non-zero first at the surface, at $H_{c 3}(T)$, while the bulk of the sample remains in the normal state until the upper (for the bulk) critical field $H_{c 2}(T)$ is reached. Early studies of surface superconductivity were on bulk samples with a flat surface so that one can easily align the sample surface with the magnetic field ${ }^{3,4}$. The nature of the vortex matter physics in the bulk between $H_{c 2}(T)$ and $H_{c 3}(T)$ has been a subject of interest ${ }^{5,6}$.

Surprisingly, it was discovered that surface superconductivity can be found in thin films when the magnetic field is applied perpendicular to the film ${ }^{7,8}$. On the other hand, if we go back to the original SJdG argument that the conditions for surface superconductivity are linearized Ginzburg-Landau equation with a simple boundary condition that the Cooper pairs cannot flow out of the sample:

$$
\left[\left(-i \hbar \frac{\partial}{\partial x}-\frac{2 e A_{x}}{c}\right) \psi\right]_{x=0}=0
$$

where $A_{x}$ is the vector potential of the magnetic field along the $x$-axis, the magnetic field $H$ is along the $z$-axis. In the original SJdG model ${ }^{1}$, the boundary condition was set at $x=0$, the $y-z$ plane, and the system of interest is in $x<0$. In fact, the SJdG theory, eq.(1), still works if the boundary is simply a thin film with a straight edge. For example, the film is in the $x-y$ half plane, $x<0$, the magnetic field is along the $z$-axis, the film edge is at $x=0, x>0$ is vacuum. Rightfully, Werner et al. ${ }^{7}$ called their effect the edge superconductivity.

It was suggested ${ }^{8}$ that much of the broadening in the resistive transition from normal state to superconducting state in a magnetic field is due to the edge superconductivity. This striking conclusion calls for a careful re-examination of the magneto-transport measurements of thin-film superconductors. Recent theoretical efforts ${ }^{9,10}$ have extended beyond the SJdG limit, to include surface roughness and disorder. With these motivations, we report a transport experiment on $\mathrm{Nb}$ thin films to investigate the longitudinal resistive transition and the Hall effect, two most important transport quantities associated with the superconducting-normal transition. The Hall effect in the superconducting-normal transition in a magnetic field has been a controversial subject ${ }^{11-21}$ with regard to the sign of the Hall coefficient and how it may change as a function of magnetic field or temperature. Our results suggest that the well-known anomaly of sign reversal in the Hall effect may in fact be caused by the edge superconductivity. 


\section{Results and discussion}

In our experiment, $\mathrm{Nb}$ thin films are used such that our findings can be directly compared with previously reported results $8,14,20$. Fig. 1 shows the representative SEM micrographs of a finished Hall bar device. As shown in Fig. 1(a), the dimensions of Hall bar region are $1 \mathrm{~mm}$ in length, $10 \mu \mathrm{m}$ in width, and the voltage probes have a width of $5 \mu \mathrm{m}$. The films are of densely packed crystalline grains of sizes less than $50 \mathrm{~nm}$ (Fig.1(d)), consistent with other polycrystalline $\mathrm{Nb}$ films ${ }^{22}$.

Fig. 2 shows our main results of the resistive transition as a function of magnetic field $H$ in our $\mathrm{Nb}$ sample, at $T=4.0 \mathrm{~K}$, and at three different angles, as shown in the inset of Fig.2(a). The longitudinal resistance $R_{x x}$ and transverse resistance $R_{x y}$ are defined as $R_{x x}=V_{x x} / I_{x}, R_{x y}=V_{x y} / I_{x}$, respectively. Since the voltage signals due to the Hall effect change sign when the magnetic field direction is reversed, we plot both the symmetric and anti-symmetric parts of the $R_{x x}$ and $R_{x y}$, as shown in Fig.2(a-d).

Fig.2(a) shows the symmetric part of $\left(R_{x x}, R_{x x}(H)+R_{x x}(-H)\right) / 2$, which is the longitudinal resistance along the $1 \mathrm{~mm}$ long path. The transition from zero resistance superconducting state to the normal state is broad, over 4000 Oe. We defined $H_{c 2}$ as the steepest slope in $R_{x x}(H) v s . H$ and $H_{c 3}$ as where the longitudinal resistance first starts to decrease from the normal state value.

Fig.2(b) shows the anti-symmetric part of $\left.R_{x x}, R_{x x}(H)-R_{x x}(-H)\right) / 2$. This quantity should be zero if the sample geometry is perfect, i.e. the voltage probes are point-like. Instead, it exhibits peaks near $H_{c 2}$, and slowly tapers off and vanishes at $H_{c 3}$. This effect is especially pronounced when the sample is rotated by a small angle $10^{\circ}$. The no-rotation configuration is when the magnetic field is perpendicular to the film (to within $1-2^{\circ}$ ).

The Hall resistance is the anti-symmetric part of $\left.R_{x y}, R_{x y}(H)-R_{x y}(-H)\right) / 2$, as shown in Fig.2(c). When the magnetic field is perpendicular to the film surface, with decreasing field, the Hall resistance is a linear function of the magnetic field down to $H_{c 2}$ where it drops to zero, with no sign change. However, when the sample is rotated by $10^{\circ}$, either around $\mathrm{x}$-axis or $\mathrm{y}$-axis, (see Fig.1(a) inset for clarity), there are oscillatory behavior in the Hall resistance starting at $H_{c 3}$. The Hall resistance clearly changes sign at $H_{c 2}$. Similar oscillatory behavior in $R_{x y}$ was reported in ${ }^{20}$. The symmetric part of the transverse resistance, shown in Fig.2(d), shows large oscillations as a function of magnetic field for all three field configurations. Later we will discuss the implications of these puzzling results.

The measurement current in Fig. 2 was $100 \mu \mathrm{A}$, the smallest current that still gives a low-noise measurement of the longitudinal and transverse resistance. Most of the features in Fig. 2 remain for currents up to $500 \mu$ A. Fig. 3 shows the effect of applied current in the $R_{x x}$ and $R_{x y}$ measurements. As shown in Fig.3(a), the broadened nature of the resistive transition is visible up to $500 \mu \mathrm{A}$, with shifts to features at $H_{c 2}$ and $H_{c 3}$. At $1 \mathrm{~mA}$, the resistance drop at $H_{c} 2$, shifted downward by about $1.5 \mathrm{kOe}$, is a large drop to zero resistance. The resistance tail due to the surface superconductivity is visible up to $H_{c 3}$, the dashed line. The oscillatory and sign change behaviors in Fig.2(c,d) disappeared. In Fig.3(b), the large negative peak in the symmetric part of $R_{x y}$ is still visible even at $1 \mathrm{~mA}$. In Fig.3(c), the sign reversal effects in the Hall resistance is largely gone.

Next, we wish to see if one can identify the bulk (interior) and the surface superconducting transitions in the temperature dependent measurements. In Fig.4(a), we converted the longitudinal resistance to resistivity $\rho_{x x}$ and plotted vs. temperature. There are indeed clear signatures of two transitions. With decreasing temperature in a constant magnetic field, the sample resistivity first deviates from the normal state value (when zoom into the $\rho_{x x}(T)$ curve one can identify fluctuation effects) around $T_{c 3}(H)$. At $T_{c 2}(H)$, the resistivity drops to zero sharply. At $I=1 \mathrm{~mA}$, it was clear in Fig. 3 that there is a small but measurable effect in the transition field, this should result in about a change (downward) of $0.7 \mathrm{~K}$ in the $T_{c}(H)$ determination. The measured $H_{c 2}$ and $H_{c 3}$ are summarized in Fig.4(b).

As shown in Fig.4(b), the location of $T_{c 2}(H)$ or $H_{c 3}(T)$ is much higher when measured at a smaller current. We suspect that the true $H_{c 3}(T)$ line determined using infinitesimally small current is even higher. At $100 \mu \mathrm{A}$, the $H_{c 3}(T)$ intercepts the $H_{c 2}(T)$ line. This is a real feature that was also observed in a Nb crystal with weak disorder ${ }^{5}$. It implies that the edge surface has a zero-field transition temperature lower than the zero-field transition temperature of the interior of the film. This is likely due to the ion damage during the IBE process, which is in fact visible in the close-up SEM micrograph in Fig.1(d).

In summary of the experimental facts: (1) We confirmed that there is indeed an edge surface superconductivity effect in thin films of $\mathrm{Nb}$ in the resistive transition in both field and temperature dependent measurements of the longitudinal resistance, as found previously ${ }^{7,8}$. (2) We found that the sign reversal phenomenon of the Hall effect is extremely sensitive to the orientation of the film device relative to the applied magnetic field. The sign reversal of the Hall coefficient appears only when the film is tilted relative to the magnetic field. When the film is perpendicular to the film, the sign-reversing Hall effect disappears. (3) When the film device is slightly tilted, large oscillations appear in the symmetric and anti-symmetric parts of the transverse resistance.

Having established the connection between the anomalous sign reversal in the Hall coefficient, we wish to finally turn our attention to the oscillations in the anti-symmetric part of $R_{x x}$ and the symmetric part of $R_{x y}$. Ideally, if the width of the voltage probes are reduced to zero, i.e. becoming a point, one should not see the anti-symmetric signals in $R_{x x}$, in Fig.2(b), and the symmetric signals in $R_{x y}$, in Fig.2(d). The fact that the voltage probes are of finite size, $5 \mu \mathrm{m}$ in this experiment, we argue that these anomalous oscillation effects may well be the Aharonov-Bohm interference effect predicted theoretically ${ }^{9,23}$. For clarity, 
the anti-symmetric (Hall effect) and the symmetric (resistive) parts of $R_{x y}$ data in Fig.3 are replotted in an expanded view in Fig.5. As shown in Fig.5, with decreasing field, when $H_{c 3}$ is crossed, the anti-symmetric part of $R_{x y}$ shows the normal state Hall effect and drops to zero at $H_{c 2}$, while the symmetric part of $R_{x y}$ exhibits a large increase at low currents $100-200 \mu \mathrm{A}$, this effect is suppressed at $500 \mu \mathrm{A}$, and a large negative peak at $H_{c 2}$.

Here we propose a tentative model for how these anomalous transport properties may arise. Even though our $\mathrm{Nb}$ thin film is very thin, $120 \mathrm{~nm}$ in thickness, the IBE process creates sharp edge surfaces in the device. As illustrated in the inset of Fig.2(a), when the magnetic field is perpendicular to the film, all of these side surfaces are parallel to the magnetic field, creating a perfect condition for the SJdG mechanism ${ }^{1}$. With decreasing magnetic field, at $H_{c 3}(T)$, the edge surface becomes superconducting first. As such, a diamagnetic supercurrent will appear at the edges, as shown by the dashed lines in Fig.5. In a transport measurement, when an external current is applied, there will be interplay between the applied current, and the spontaneously formed supercurrent. The latter has a topological feature similar to the edge currents in the integer quantum Hall effect ${ }^{24-26}$. Both are due to the orbital motion of the charged carriers coupled to the vector potential of the magnetic field (Landau orbits), they cannot flow out of the boundary, and are phase coherent (albeit for different physical mechanisms). The top and bottom panels in Fig.5 are for opposite field directions, the top for field into the page, and bottom for out of the page. The applied current is from left to right. When the magnetic field is reversed, the surface supercurrent (blue dashed lines) will reverse its direction (to remain diamagnetic). Since the voltage probes are fixed, A-B for the longitudinal voltage and B-C for the transverse voltage, the potential drops between A-B and B-C will depend on the field direction since the applied current and the edge supercurrent are additive in the top panel, but in subtraction in the bottom panel. When the applied current is small, it will also flow as Cooper pairs along the same paths as the diamagnetic screening supercurrent, namely the blue dashed lines and the red lines in Fig.5 are all on the edge surfaces, and they are all phase coherent and sensitive to the magnetic flux their closed paths enclose. Thus when the magnetic field is changed, the Aharonov-Bohm interference effects will occur between these currents. This current interference effect will manifest as resistance oscillations similar to multi-connected wire networks ${ }^{27}$. The novelty here is that the interference oscillations appear in a singly connected superconductor.

\section{Methods}

\section{Sample preparation and characterization.}

The $\mathrm{Nb}$ thin films used in this experiment have a thickness of $120 \mathrm{~nm}$, deposited on $\mathrm{SiO}_{2} / \mathrm{Si}$ substrates by DC magnetron sputtering. The details of the deposition process can be found elsewhere ${ }^{28,29}$. Our Nb films do not show aging effect, there is no measurable change in the transition temperature over time (days). This is not surprising since aging effect was known to occur in $\mathrm{Nb}$ films with thickness at below $50 \mathrm{~nm}^{30}$.

The zero-field superconducting transition temperature $T_{c}$ of the $\mathrm{Nb}$ film was measured to be $8.85 \mathrm{~K}$. The residual resistivity ratio $(R R R) \rho_{x x}(300 \mathrm{~K}) / \rho_{x x}(10 \mathrm{~K})$ is 4.3 . The $10 \mathrm{~K}$ resistivity of our sample is $4.2 \mu \Omega \mathrm{cm}$. The carrier density $n$ is measured to be $6.5 \times 10^{22} \mathrm{~cm}^{-3}$ according to the Hall resistance formula $R_{x y}=B /($ nte $)$ above $T_{c}$ where $t$ is the film thickness $(B=H$ in the CGS unit we adopt here). The Fermi wavevector is $k_{F}=\left(3 \pi^{2} n\right)^{1 / 3}=1.25 \AA^{-1}$.

It should be noted that the Hall coefficient of $\mathrm{Nb}$ is positive (hole-like) in the normal state due to its "jungle-gym" shaped Fermi surface ${ }^{31-36}$. This is what we found here as well. The Drude mean free path, $l=\frac{\hbar k_{F}}{n e^{2} \rho_{x x}}$, of the carriers at $10 \mathrm{~K}$ is about $18.5 \mathrm{~nm}$. The upper critical field $H_{c 2}$ at $4 \mathrm{~K}$ is $12.2 \mathrm{kOe}$. Using $H_{c 2}=\frac{\phi_{o}}{2 \pi \xi^{2}}$, we obtain a coherence length $\xi=16.3 \mathrm{~nm}$. In comparison, the upper critical field $H_{c 2}(4 K)=4.3 \mathrm{kOe}$ was found for a $\mathrm{Nb}$ crystal with weak disorder ${ }^{5}$, giving a coherence length of $27.5 \mathrm{~nm}$. For an annealed $\mathrm{Nb}$ crystal with a $R R R=1550^{31}$, the upper critical field at $4.2 \mathrm{~K}$ was $2.6 \mathrm{kOe}$, giving a coherence length of $73 \mathrm{~nm}$. Since $\xi^{-1}=\xi_{o}{ }^{-1}+l^{-1}$, where $\xi_{o}$ is the intrinisic coherence length, thus the coherence length in our $\mathrm{Nb}$ film is limited by the gain boundary scattering of the carriers.

\section{Device fabrication and transport measurement .}

Our Nb films were patterned into Hall bar devices using photolithography and an ion beam etching (IBE) process ${ }^{37}$. The IBE process utilizes the physical process of ion ballistic collision, and it results in sharp edges in the devices ${ }^{37}$.

The temperature dependent longitudinal resistances $R_{x x}$ and transverse resistances $R_{x y}$ of the $\mathrm{Nb}$ film device were measured by the standard four-probe method using the Physical Property Measurement System (PPMS-9, Quantum Design Inc.). The low resistance contacts were made using silver paste (Dupont 5025 Conductor Paste). The voltages were measured using AC resistance option within the PPMS, with alternating current directions to eliminate thermal electric signals. For Hall effect, the measurements were carried out in both $+H$ and $-H$ directions such that one can obtain both symmetric and anti-symmetric parts of the longitudinal and transverse resistances (or conductances). In the fixed $T$ measurements, the sample temperature is regulated to within $20 \mathrm{mK}$ of the set temperature. In the fixed $H$ measurements, the field in the superconducting magnet remains constant. 


\section{References}

1. Saint-James, D. \& de Gennes, P. G. Onset of superconductivity in decreasing fields. Phys. Lett. 7, 306-308 (1963).

2. Tinkham, M. Introduction to superconductivity (McGraw-Hill, New York, 1996, chapter 4).

3. Hempstead, C. F. \& Kim, Y. B. Resistive transitions and surface effects in type-II superconductors. Phys. Rev. Lett. 12, 145-148 (1964).

4. de la Cruz, F., Maloney, M. D. \& Cardona, M. Variation of the ratio $\frac{H_{c 3}}{H_{c 2}}$ in the immediate vicinity of $T_{c}$. Phys. Rev. 187, 766-767 (1969).

5. Park, S. R., Choi, S. M., Dender, D. C., Lynn, J. W. \& Ling, X. S. Fate of the peak effect in a type- II superconductor: Multicriticality in the bragg-glass transition. Phys. Rev. Lett. 91, 167003 (2003).

6. Das, P. et al. Surface superconductivity, positive field cooled magnetization, and peak-effect phenomenon observed in a spherical single crystal of niobium. Phys. Rev. B 78, 214504 (2008).

7. Werner, R. et al. Edge superconductivity in $\mathrm{Nb}$ thin film microbridges revealed by electric transport measurements and visualized by scanning laser microscopy. Supercond. Sci. Technol. 26, 95011-95021(11) (2013).

8. Zeinali, A., Golod, T. \& Krasnov, V. M. Surface superconductivity as the primary cause of broadening of superconducting transition in Nb films at high magnetic fields. Phys. Rev. B 94, 214506 (2016).

9. Xie, H. Y., Kogan, V. G., Khodas, M. \& Levchenko, A. Onset of surface superconductivity beyond the Saint-James-de Gennes limit. Phys. Rev. B 96, 104516 (2017).

10. Croitoru, M. D., Shanenko, A. A., Chen, Y., Vagov, A. \& Albino Aguiar, J. Microscopic description of surface superconductivity. Phys. Rev. B 102, 054513 (2020).

11. Zhao, S. Y. F. et al. Sign-reversing hall effect in atomically thin high-temperature $\mathrm{Bi}_{2.1} \mathrm{Sr}_{1.9} \mathrm{CaCu}_{2.0} \mathrm{O}_{8+\delta}$ superconductors. Phys. Rev. Lett. 122, 247001 (2019).

12. Ao, P. Comment on "sign-reversing hall effect in atomically thin high-temperature $\mathrm{Bi}_{2.1} \mathrm{Sr}_{1.9} \mathrm{CaCu}_{2.0} \mathrm{O}_{8+\delta}$ superconductors". Phys. Rev. Lett. 124, 249701 (2020).

13. Zhao, S. Y. F. et al. Zhao et al. reply:. Phys. Rev. Lett. 124, 249702 (2020).

14. Hagen, S. J., Lobb, C. J., Greene, R. L. \& Eddy, M. Flux-flow Hall effect in superconducting $\mathrm{Tl}_{2} \mathrm{Ba}_{2} \mathrm{CaCu}_{2} \mathrm{O}_{8}$ films. Phys. Rev. B 43, 6246-6248 (1991).

15. Dorsey, A. T. \& Fisher, M. P. A. Hall effect near the vortex-glass transition in high-temperature superconductors. Phys. Rev. Lett. 68, 694-697 (1992).

16. Kopnin, N. B., Ivlev, B. I. \& Kalatsky, V. A. The flux-flow hall effect in type II superconductors. an explanation of the sign reversal. J. Low Temp. Phys 90, 1-13 (1993).

17. Wang, Z. D., Dong, J. \& Ting, C. S. Unified theory of mixed state Hall effect in type-II superconductors: Scaling behavior and sign reversal. Phys. Rev. Lett 72, 3875 (1994).

18. Chen, J. L. \& Yang, T. J. Anomalous Hall effect from vortex motion in high- $T_{c}$ superconductors. Phys. Rev. B 50, 4064 (1994).

19. Ao, P. Motion of vacancies in a pinned vortex lattice: origin of the Hall anomaly. J.Phys. Condens. Matter 10, L677-L682 (1998).

20. Ghenim, L. et al. Transport and vortex pinning in micron-size superconducting Nb films. Phys. Rev. B 69, 064513 (2004).

21. Nakai, N., Hayashi, N. \& Machida, M. Direct numerical confirmation of pinning-induced sign change in the superconducting Hall effect in type-II superconductors. Phys. Rev. B 83, 024507 (2011).

22. Benvenuti, C. et al. Structural and rf properties of niobium films deposited onto annealed niobium resonators. Proc. 1997 Work. on RF Supercond. Abano Terme (Padova), Italy 4, 1065-1073 (1997).

23. Aleiner, I. L., Andreev, A. V. \& Vinokur, V. Aharonov-Bohm oscillations in singly connected disordered conductors. Phys. Rev. Lett. 114, 076802 (2015).

24. Halperin, B. I. Quantized hall conductance, current-carrying edge states, and the existence of extended states in a two-dimensional disordered potential. Phys. Rev. B 25, 2185-2190 (1982).

25. Creff, M., Faisant, F., Rubi, J. M. \& Wegrowe, J. E. Surface currents in hall devices. J. Appl. Phys. 128, 054501 (2019).

26. Cage, M. E. Current distributions in quantum Hall effect devices. J. Res. Natl. Inst. Stand. Technol. 102, 677-691 (1997). 
27. Ling, X. S. et al. Nature of Phase Transitions of Superconducting Wire Networks in a Magnetic Field. Phys. Rev. Lett. 76, 2989 (1996).

28. Zhang, W. J. et al. Crossover behaviors in magnetoresistance oscillations for Nb thin film with rectangular arrays of antidots. Eur. Lett. 99, 37006 (2012).

29. He, S. K. et al. Wire network behavior in superconducting nb films with diluted triangular arrays of holes. J. Phys. Condens. Matter 24, 155702 (2012).

30. Santavicca, D. F. \& Prober, D. E. Aging of Ultra-Thin Niobium Films. IEEE Trans. Appl. Supercond. 25, 1-4 (2015).

31. Reed, W. A., Fawcett, E. \& Kim, Y. B. Observation of the hall effect in superconductors. Phys. Rev. Lett. 14, 790 (1965).

32. Berlincourt, T. G. Hall effect, resistivity, and magnetoresistivity of Th, U, Zr, Ti, and Nb. Phys. Rev 114, 969 (1959).

33. Fawcett, E., Reed, W. A. \& Soden, R. R. High-field galvanomagnetic properties of niobium and tantalum. Phys. Rev. 159, 533 (1967).

34. Mattheiss, L. F. Electronic structure of niobium and tantalum. Phys. Rev. 1, 373 (1970).

35. Halloran, M. H., Condon, J. H., Graebner, J. E., Kunzier, J. E. \& Hsu, F. S. L. Experimental study of the fermi surface of niobium and tantalum. Phys. Rev. B 1, 366 (1970).

36. Seng, P., Logvenov, G. Y., Tidecks, R., Samwer, K. \& Oboznov, V. Hall effect in superconducting Nb/Cu multilayers. Phys. C 235-240, 2565-2566 (1994).

37. Yoshida, T. \& Tsuge, H. Novel josephson integrated circuit technology based on selective trilayer ion-beam etching process. l7th Conf. on Solid State Devices Mater. 127-130, DOI: 10.7567/SSDM.1985.C-1-8 (1985).

LaTeX formats citations and references automatically using the bibliography records in your .bib file, which you can edit via the project menu. Use the cite command for an inline citation, e.g. .

For data citations of datasets uploaded to e.g. figshare, please use the howpublished option in the bib entry to specify the platform and the link, as in the Hao:gidmaps:2014 example in the sample bibliography file.

\section{Acknowledgements}

We wish to thank Professor Qianggang Qiu of IoP-CAS for providing the Nb thin film samples, and Professor Ping Ao of Shanghai University for urging us to investigate this problem. We acknowledge useful discussions with Professor Jinwu Ye and for his useful comments on the manuscript. This work was partially supported by National Natural Science Foundation of China, Grant No. 51772200 (PI: Tang).

\section{Author contributions statement}

D.Z., H.S.Z., R.J.T., X.S.L. and Z.H.H. design, discussed and monitored the progress of research projects. Z.W.H and Z.X.P fabricated the $\mathrm{Nb}$ thin films. D.Z. and H.S.Z. fabricated Nb film Hall bar device. D.Z. measured and analyzed the data. D.Z., R.J.T. and X.S.L. finalized the manuscript with inputs from all the authors. 

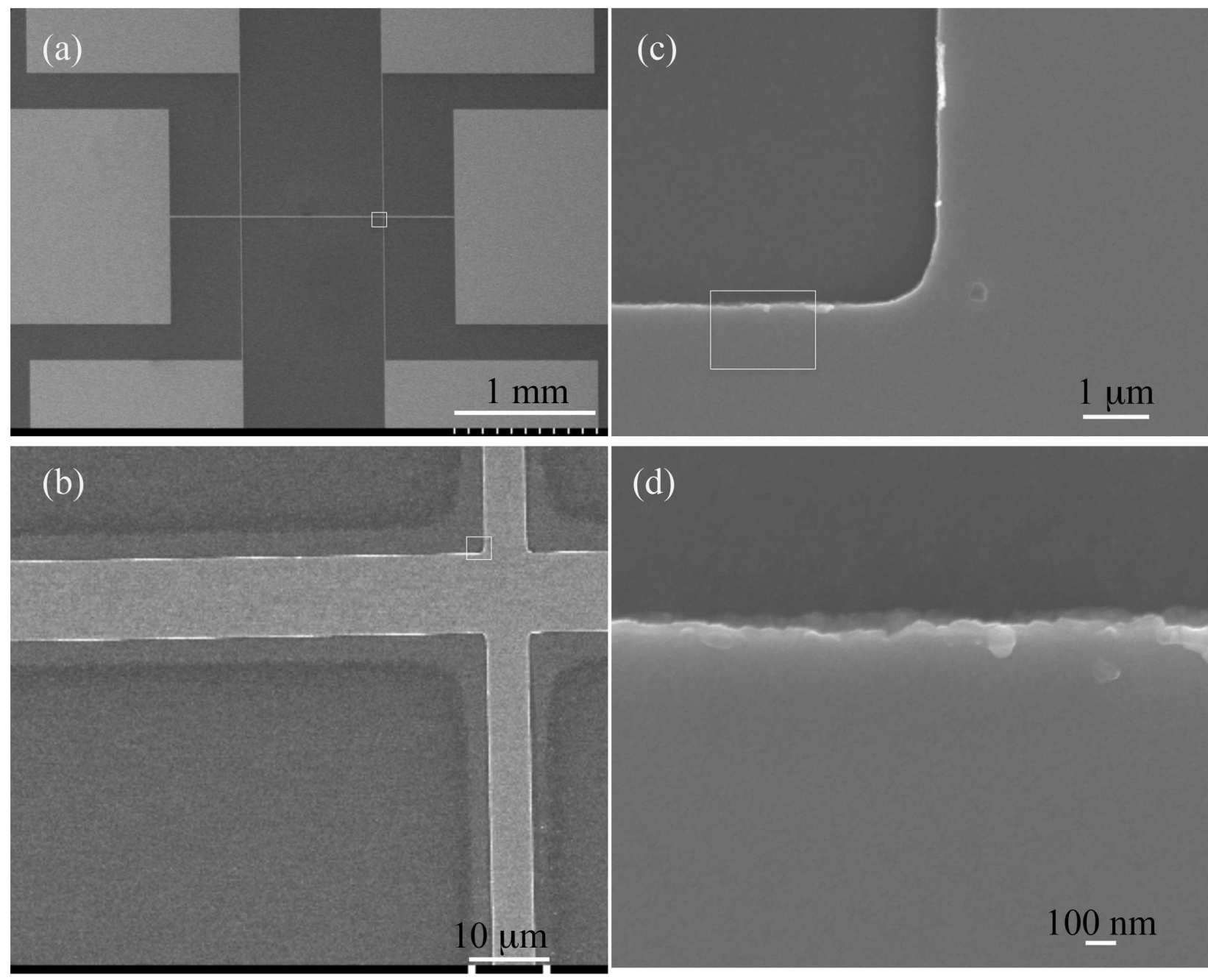

\section{(d)}

Figure 1. Scanning electron microscope (SEM) micrographs. (a) The SEM micrograph of a patterned Nb thin film sample. Scale bar: $1.0 \mathrm{~mm}$. (b) The expanded view of the small box region in (a). Scale bar: $10.0 \mu \mathrm{m}$. (c-d) close-ups with scales bars $1 \mu \mathrm{m}$ and $100 \mathrm{~nm}$, respectively. The small boxes in (a-c) are approximately the regions for expanded views in the subsequent micrographs $(b-d)$. 

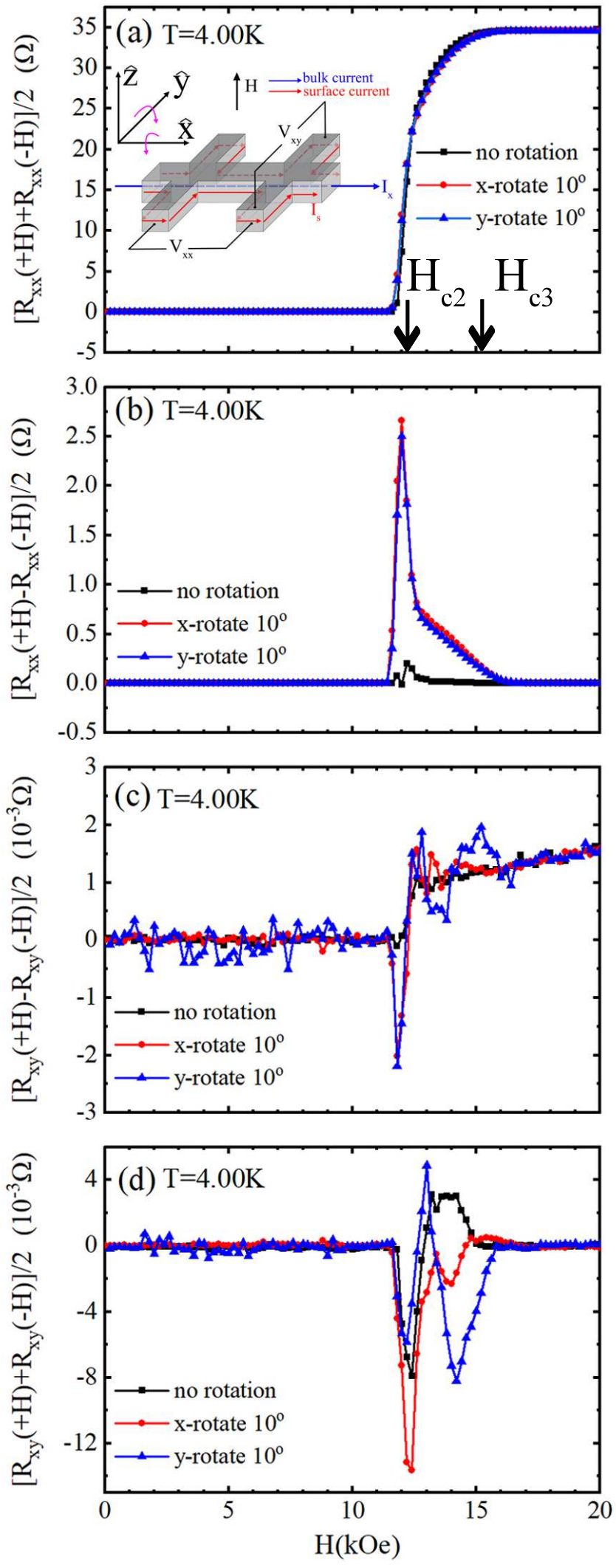

Figure 2. (Color online) Magnetic field dependence of longitudinal and transverse resistances. (a) The symmetric part of the longitudinal resistance $R_{x x}$; (b) The anti-symmetric part of the longitudinal resistance; (c) The Hall resistance, the anti-symmetric part of the transverse resistance; (d) The symmetric part of the transverse resistance. $I=100 \mu \mathrm{A}, T=4.0 \mathrm{~K}$. 

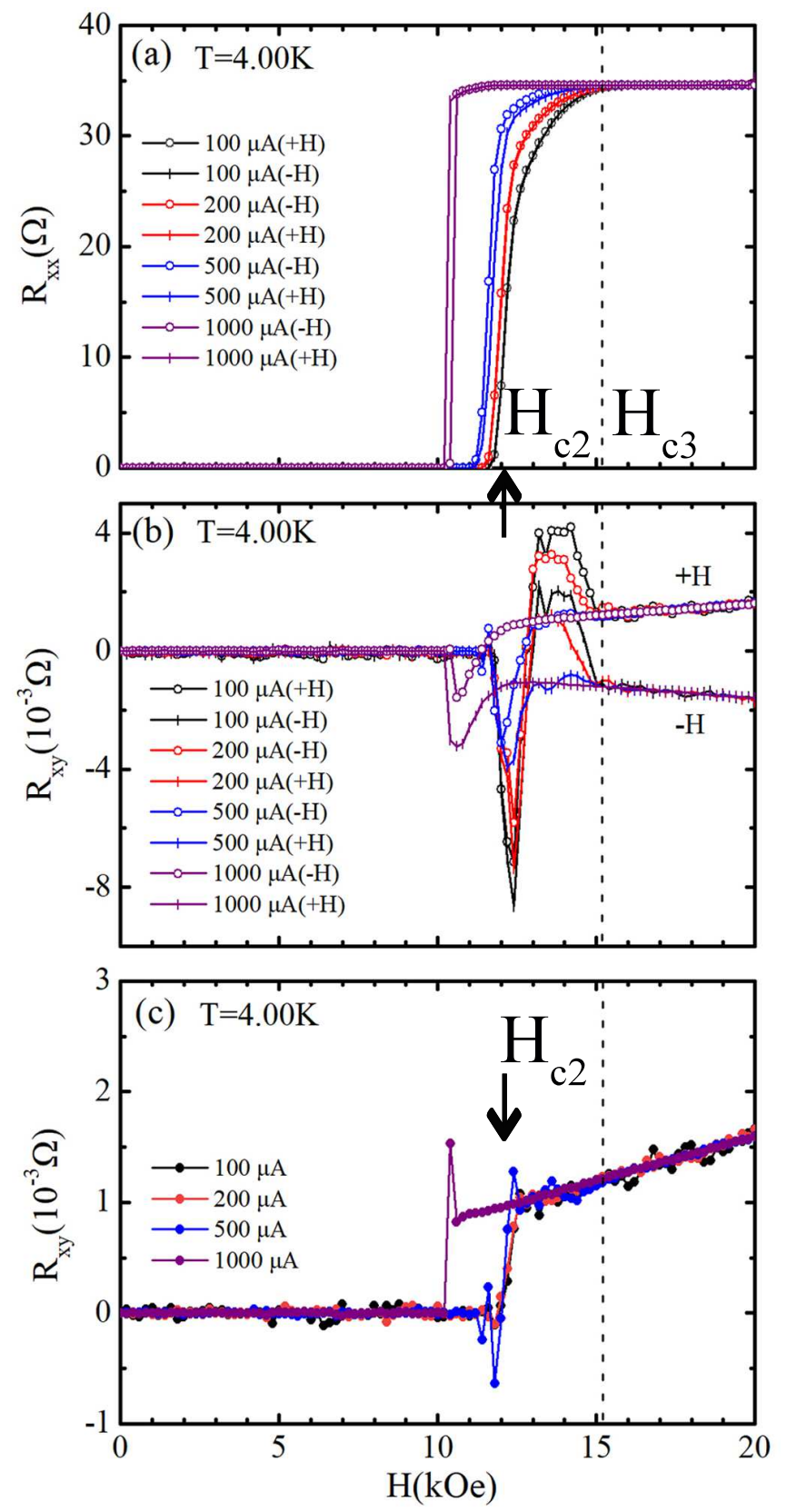

Figure 3. (Color online) Effects of applied current. (a) The longitudinal resistance $R_{x x}$ vs. $H$; (b) The transverse resistance $R_{x y}$ at $+H$ and $-H$ field directions, vs. $H$; (c) The anti-symmetric part of the transverse resistance $R_{x y}$, i.e. the Hall resistance vs. $H$. 

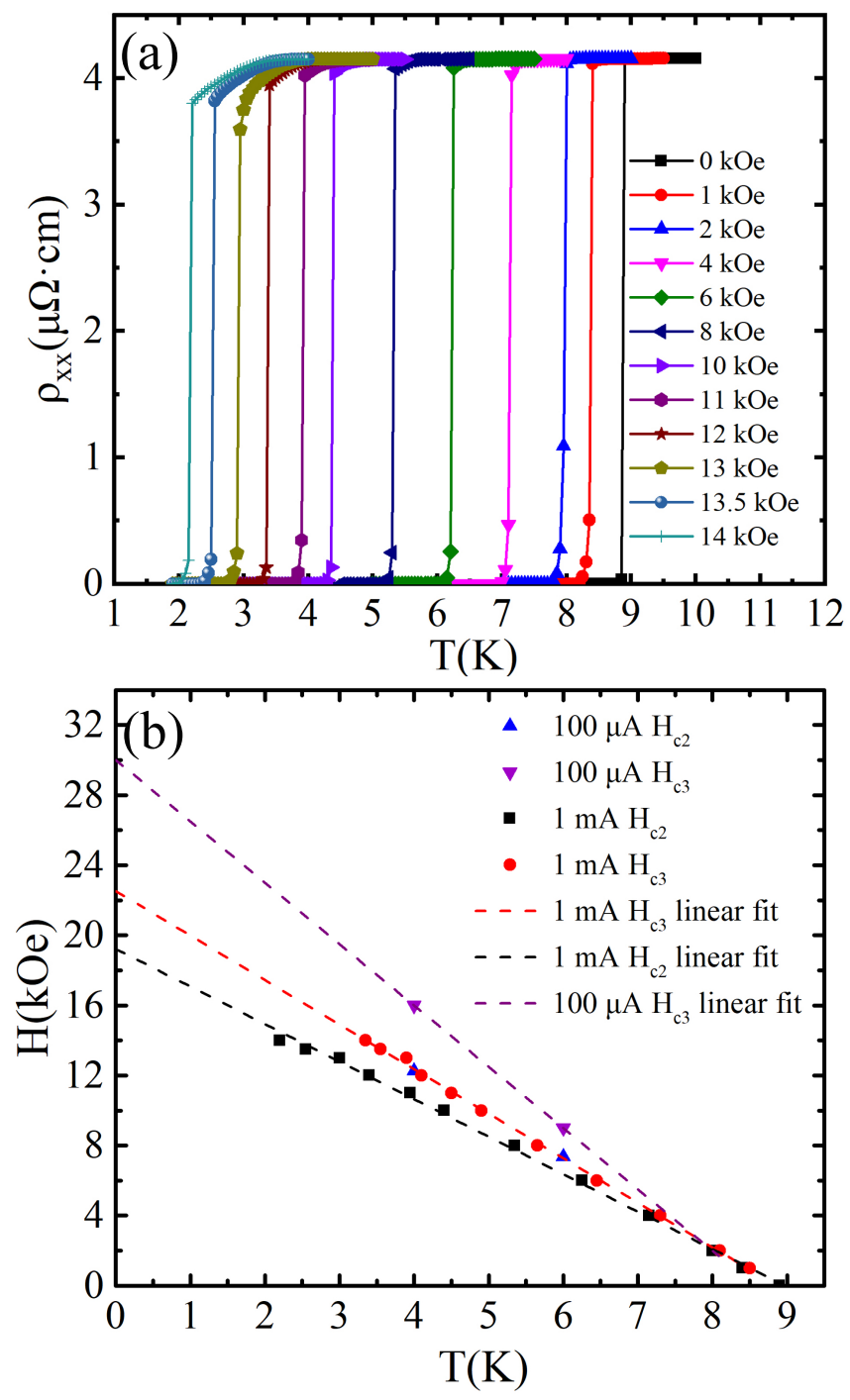

Figure 4. (Color online) Phase diagram measurements: (a) $\rho_{x x}$ vs. $T$ at different magnetic fields, $I=1 \mathrm{~mA}$. The magnetic field is perpendicular to the film, thus parallel to the edge surfaces. (b) $H-T$ phase diagram constructed using the criteria discussed in the text. 


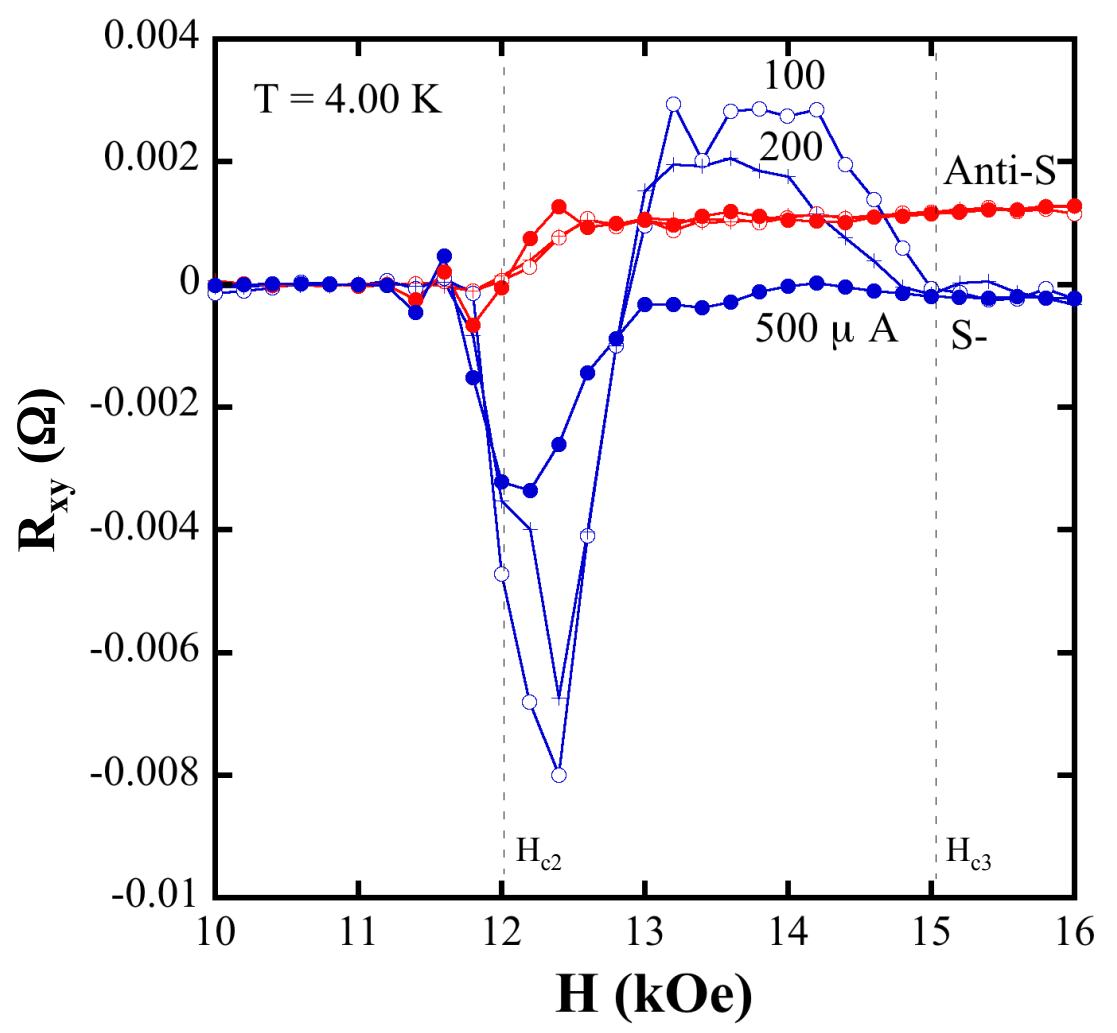

Figure 5. (Color online) Anti-symmetric (red, Hall effect) and symmetric parts (blue) of transverse resistance $R_{x y}$, calculated from Fig.3. $R_{x y}($ Anti-S $)=\left(R_{x y}(H)-R_{x y}(-H)\right) / 2, R_{x y}(\mathrm{~S})=\left(R_{x y}(H)+R_{x y}(-H)\right) / 2$. Open circles, crosses, and solid circles are for 100,200 , and $500 \mu \mathrm{A}$ respectively. The $100 \mu \mathrm{A}$ data set is the same as the "no rotation" set in Fig.2. 

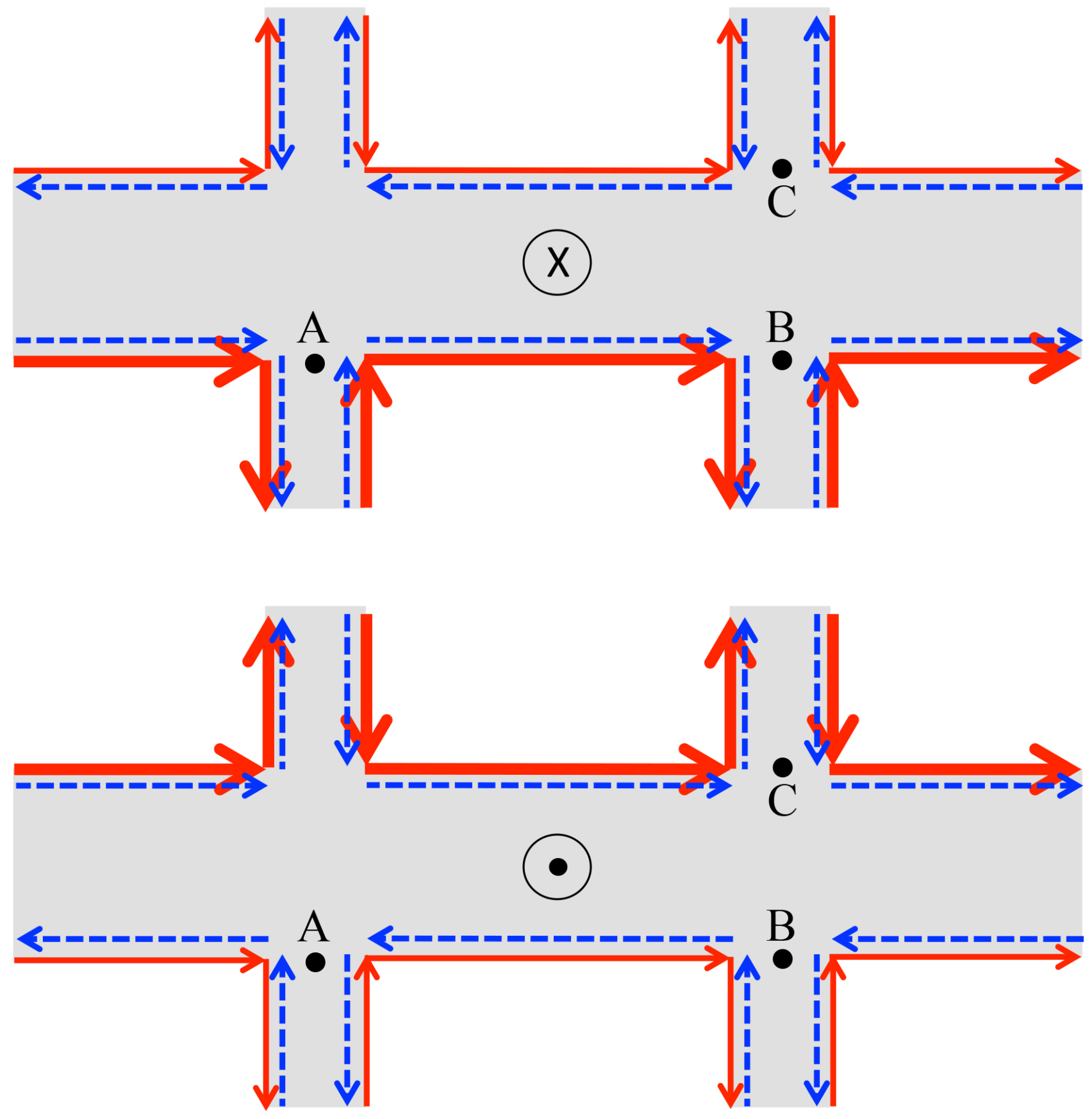

Figure 6. (Color online) A tentative model for current distribution. (Upper panel) The current distribution for magnetic field pointing into the page; (Lower panel) for magnetic field pointing out of the page. The dashed blue lines are the diamagnetic current when the edge surface first becomes superconducting by the Saint-James-de Gennes mechanism. 


\section{Figures}

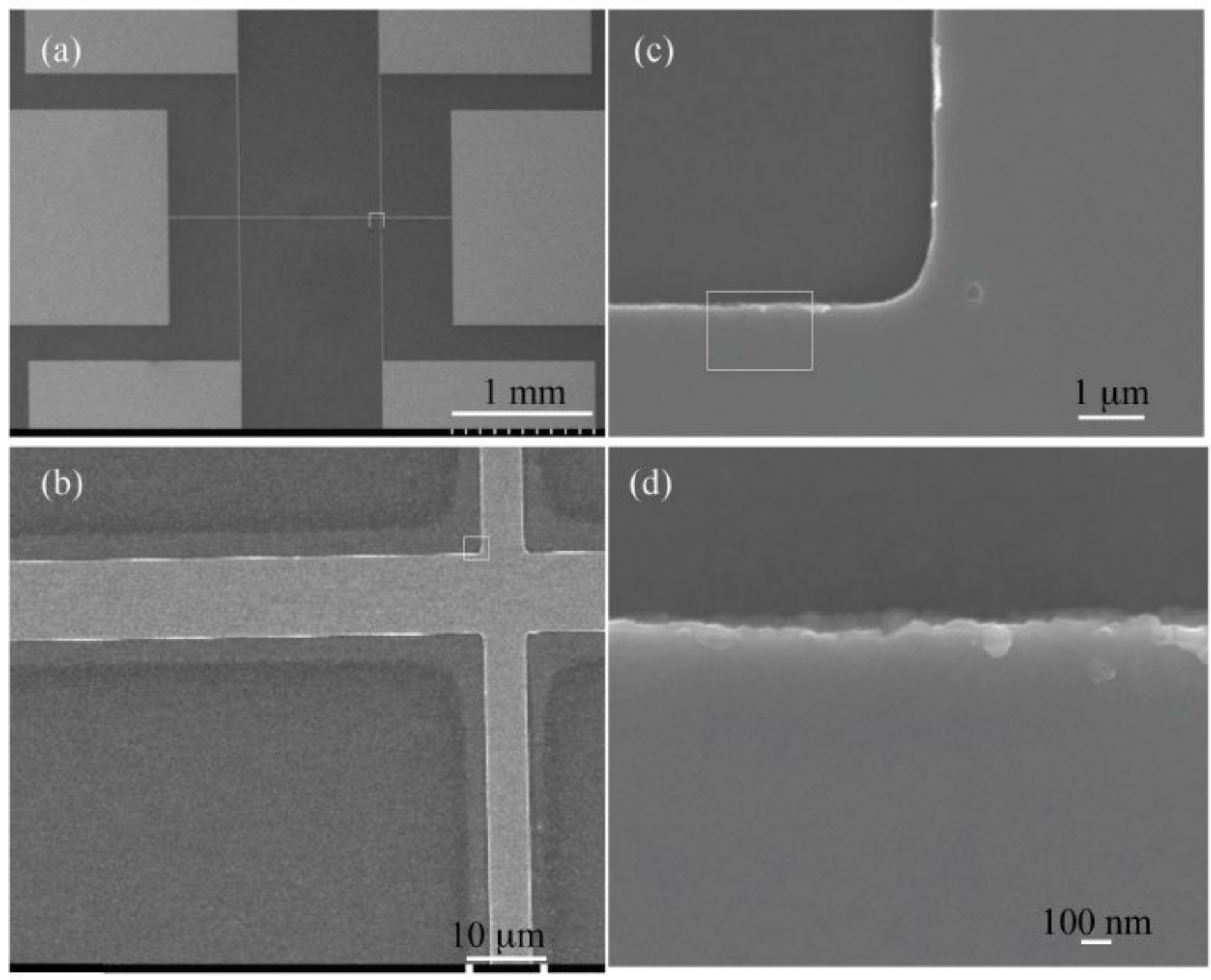

\section{Figure 1}

Scanning electron microscope (SEM) micrographs. (a) The SEM micrograph of a patterned Nb thin film sample. Scale bar: $1.0 \mathrm{~mm}$. (b) The expanded view of the small box region in (a). Scale bar: $10.0 \mathrm{~mm}$. (cd) close-ups with scales bars $1 \mathrm{~mm}$ and $100 \mathrm{~nm}$, respectively. The small boxes in (a-c) are approximately the regions for expanded views in the subsequent micrographs (b-d). 

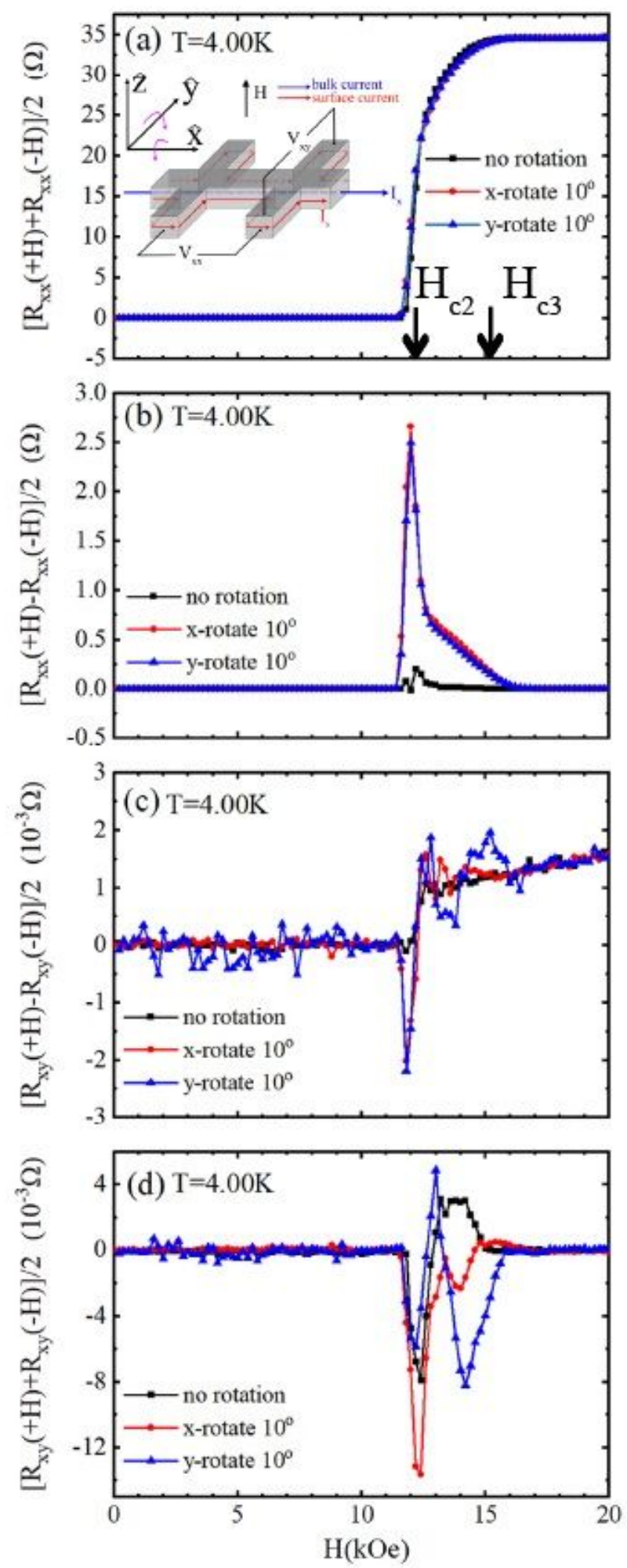

\section{Figure 2}

(Color online) Magnetic field dependence of longitudinal and transverse resistances. (a) The symmetric part of the longitudinal resistance Rxx; (b) The anti-symmetric part of the longitudinal resistance; (c) The Hall resistance, the anti-symmetric part of the transverse resistance; (d) The symmetric part of the transverse resistance. $\mathrm{I}=100 \mathrm{~mA}, \mathrm{~T}=4.0 \mathrm{~K}$. 

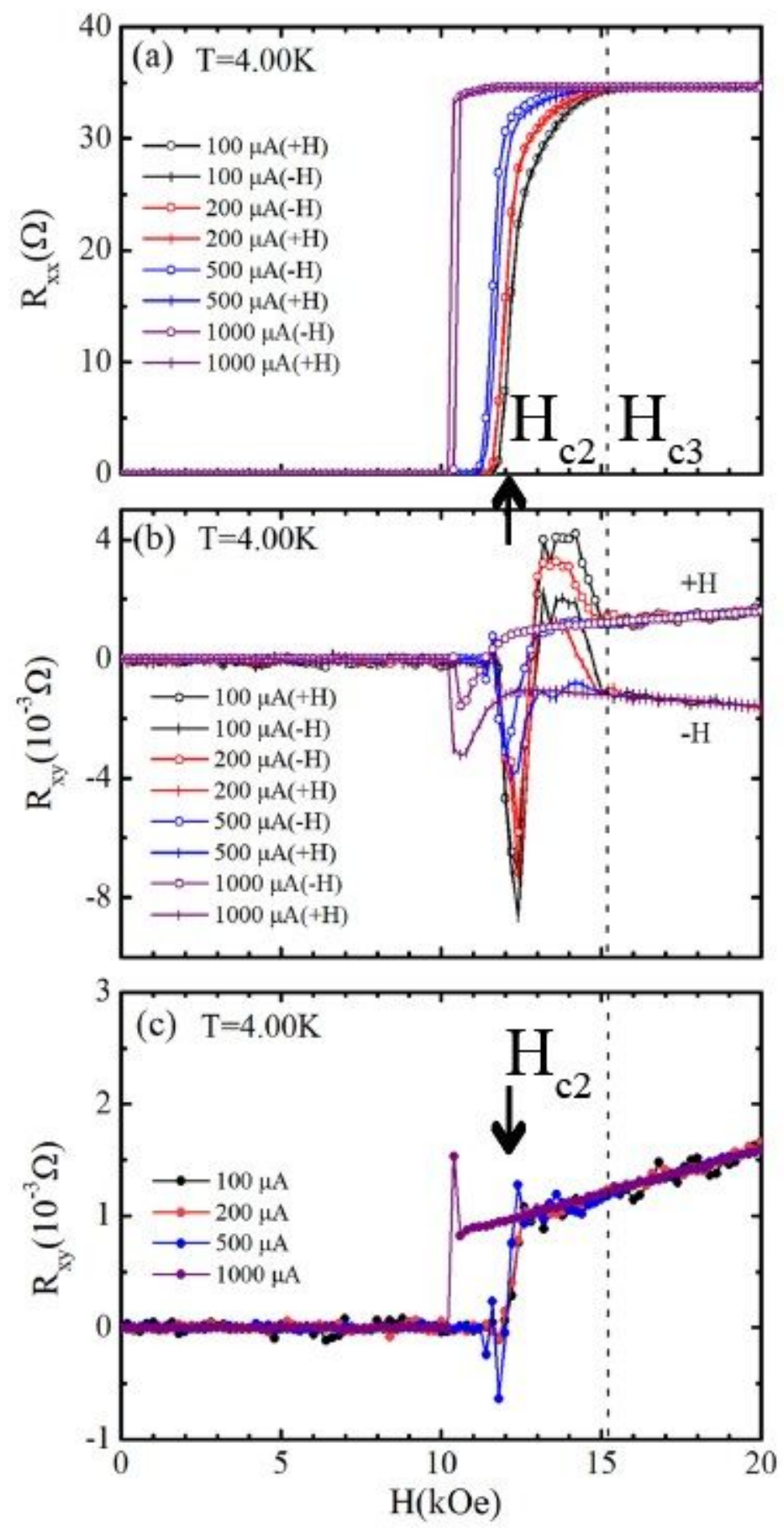

Figure 3

(Color online) Effects of applied current. (a) The longitudinal resistance Rxx vs. H; (b) The transverse resistance Rxy at $+\mathrm{H}$ and $-\mathrm{H}$ field directions, vs. $\mathrm{H}$; (c) The anti-symmetric part of the transverse resistance Rxy, i.e. the Hall resistance vs. $H$. 

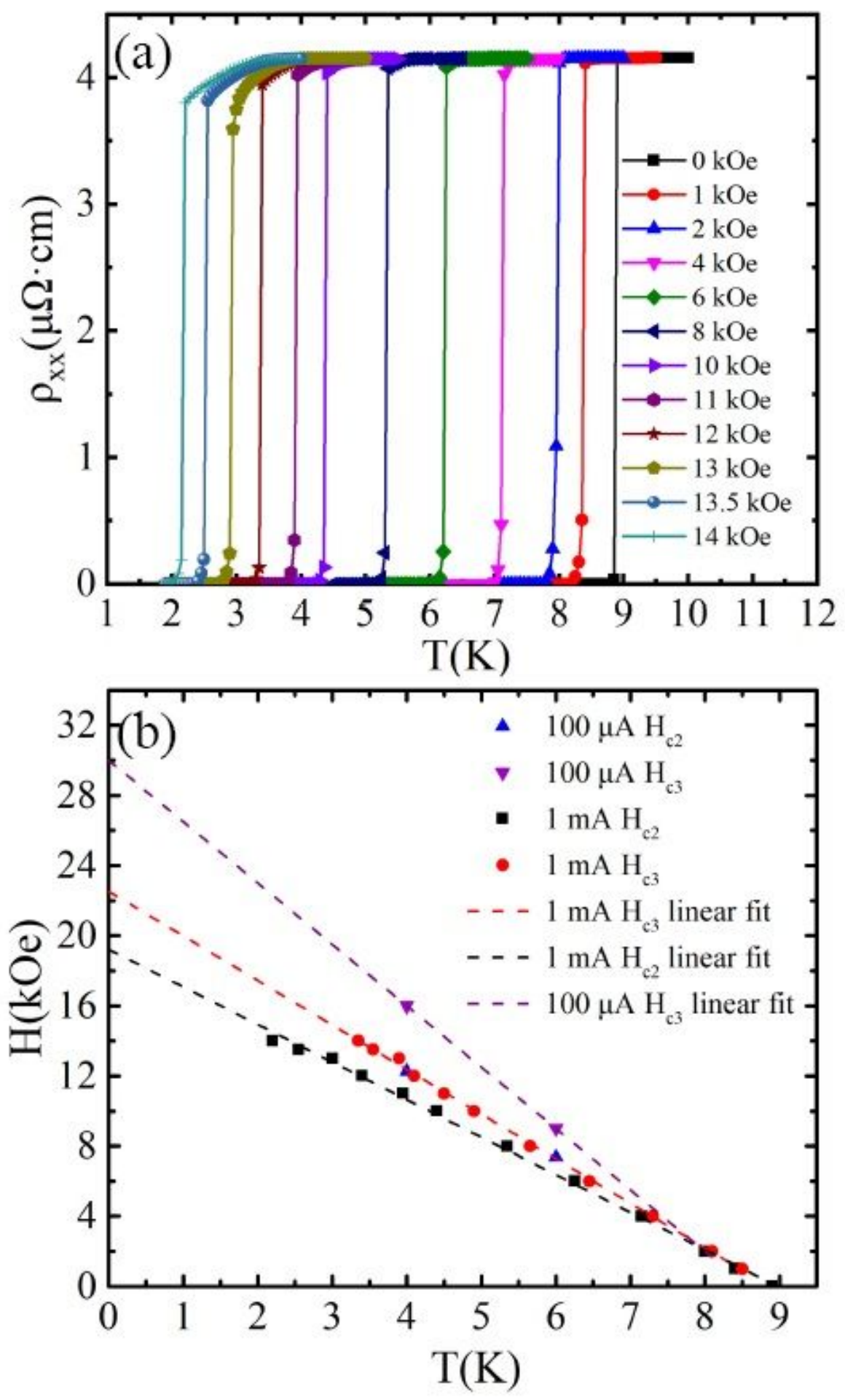

Figure 4

(Color online) Phase diagram measurements: (a) rxx vs. T at different magnetic fields, I = $1 \mathrm{~mA}$. The magnetic field is perpendicular to the film, thus parallel to the edge surfaces. (b) $\mathrm{H}$ - T phase diagram constructed using the criteria discussed in the text. 


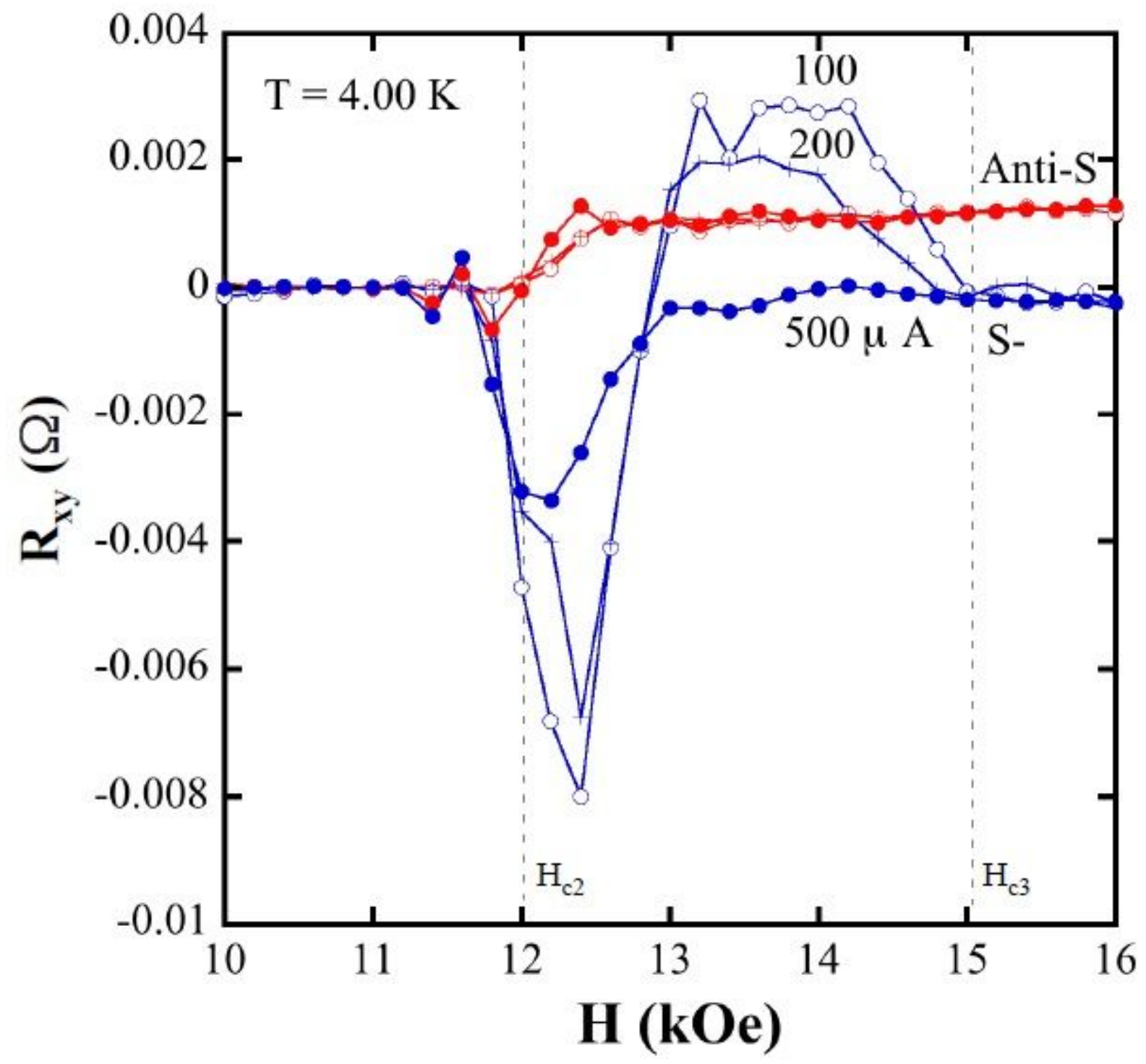

Figure 5

(Color online) Anti-symmetric (red, Hall effect) and symmetric parts (blue) of transverse resistance Rxy, calculated from Fig.3. $\operatorname{Rxy}($ Anti-S $)=(\operatorname{Rxy}(H)-\operatorname{Rxy}(-H))=2, \operatorname{Rxy}(\mathrm{S})=(\operatorname{Rxy}(\mathrm{H})+\operatorname{Rxy}(-\mathrm{H}))=2$. Open circles, crosses, and solid circles are for 100, 200, and 500mA respectively. The $100 \mathrm{~mA}$ data set is the same as the "no rotation" set in Fig.2. 

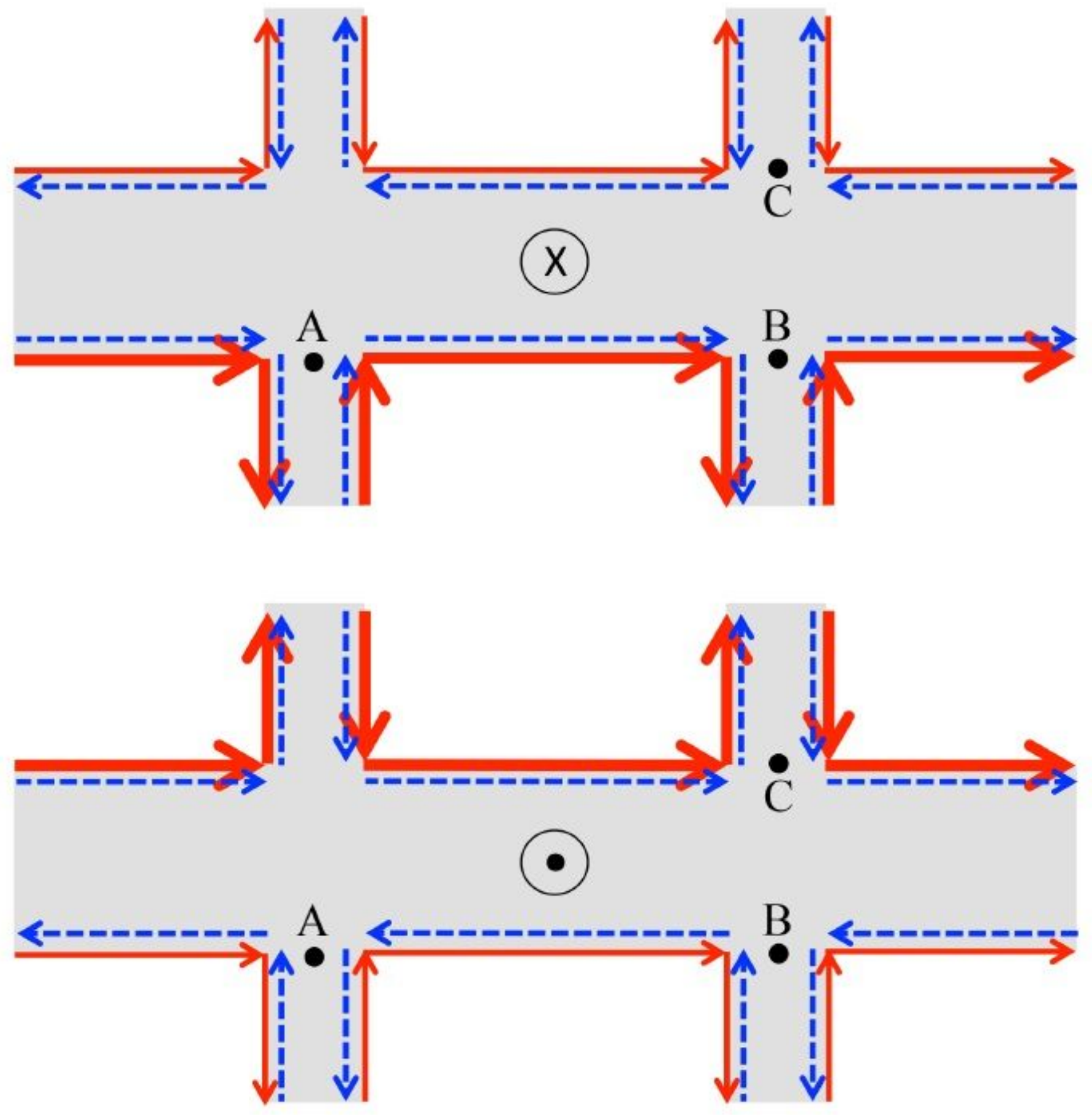

Figure 6

(Color online) A tentative model for current distribution. (Upper panel) The current distribution for magnetic field pointing into the page; (Lower panel) for magnetic field pointing out of the page. The dashed blue lines are the diamagnetic current when the edge surface first becomes superconducting by the Saint-James-de Gennes mechanism. 